\title{
REVIEW
}

\section{Thai men's health and sexual attitude}

\author{
Apichat Kongkanand ${ }^{1}$, Sompol Permpongkosol ${ }^{2}$ and Kavirach Tantiwongse ${ }^{3}$
}

Men's health awareness, including the research and study of quality of life, sexual desires and risk factors, has increased worldwide. In Thailand, this advancement is made possible by cooperation, research and sponsorship from the local Thai community. This article aims to illustrate the sexual attitudes of Thai people, to determine the degree of erectile dysfunction (ED) and to investigate how to manage and cope with ED in a Thai community. We reviewed the relevant literature from Thai-based articles and surveys in regard to men's health, sexual attitudes, the prevalence of ED and common risk factors in the Thai community. The primary risk factor for ED in Thai men was age-related health decline and the presence of vascular disease. Most Thai men will seek consultation from their partner in regard to ED. The main presentation of metabolic disease in Thai patients was dyslipidemia. New selective serotonin reuptake inhibitors are not available for premature ejaculation in Thai communities. The debate in regard to malpractice compensation is an issue that should be closely monitored. There is currently a shortage of home care for the elderly in Thailand. The insights provided by the articles helped recruit the study patients and in turn, helped us gain knowledge that can be translated into improved men's health care in Thailand.

Asian Journal of Andrology (2011) 13, 534-536; doi:10.1038/aja.2010.122; published online 13 June 2011

Keywords: erectile dysfunction; sexual attitudes; Thai men's health

\section{INTRODUCTION}

The study of men's health in Thailand was initiated over 20 years ago with a primary study population consisting of hospital-based patients. The patient recruitment methodology had various limitations because it was not a population-based survey and was thus not reflective of the Thai population. Men's health in Asia and especially in Thailand, gained significant awareness and insight over the years. Thailand has a population of 64 million people, with the majority involved in the agriculture industry. The Thai people are known for their zealous and diligent working culture that translates into an extremely high quantity of exported agriculture products. Despite having a $90 \%$ literacy rate, the prevalence of poor nutrition, inadequate rest and an unstable political environment have burdened the population over the years. Nevertheless, men's health has steadily improved, resulting in a longer lifespan than ever before. Moreover, in 1997, a breakthrough of erectile dysfunction (ED) treatment called Viagra was launched, thereby paving the way for a significant amount of men's health awareness research, including studies that evaluated quality of life, sexual desires and risk factors in Thailand and worldwide. ${ }^{1-3}$

The study of men's health was initiated as an ED prevalence study. The first study occurred in Thailand in $1998^{4,5}$ through cooperation with the Erectile Dysfunction Advisory Committee on the Treatment of Thailand and the National Institute of Development Administration; the study was supported by Pfizer (Thailand) Ltd. Pfizer (Thailand) Ltd had had no influence on the study outcomes. The results from this nationwide study, which targeted Thai men aged between 40 and 70 years, laid the foundation in regard to the understanding and development of ED research in
Thailand that could be studied and translated into improved patients' health and quality of life. The study reported an ED prevalence of 37.5\% and revealed risk factors related to ED and men's health.

In Thailand, the management and treatment of aging males are studied through a collaborative effort between various physician specialties, referred to as the Golden Age Group, which includes gynaecological surgeons, urologists and geriatric physicians. These physicians are extremely active and enthusiastic in their fields and aim to provide the best medical care for aging males. They practice primarily in major universities and community hospitals throughout the country, including Chiang Mai, Songkhla, Korat, Ubonrachatani and Nakonsawan provinces.

Through increased advertisements and awareness of male aging conditions, such as prostate diseases, hypogonadism, ED and urinary incontinence, this network of physicians has significantly increased the number of men presenting to hospital clinics with age-related male diseases or disorders. Moreover, the campaign to increase awareness of prostate cancer, which is conducted on Father's Day and, occasionally, during festivals such as Songkran (Water Festival) or Buddhist Lent, provides an opportunity to encourage patients to visit clinics where each patient can be routinely screened for prostate-specific antigen, a prostate cancer tumor marker.

\section{CURRENT OUTLOOK}

There are numerous studies evaluating men's health, the elderly and aging males. Although these various terminologies are used interchangeably, men's health appears to resonate better with patients, especially for clinical trial recruitment purposes, and includes benign prostate hyperplasia (BPH), ED, hypogonadism and premature ejaculation (PE); of

${ }^{1}$ Bumrungrad International Hospital, Bangkok, Thailand; ${ }^{2}$ Faculty of Medicine, Ramathibodi Hospital, Mahidol University, Bangkok, Thailand and ${ }^{3}$ Faculty of Medicine, Chulalongkorn University, Bangkok, Thailand

Correspondence: Dr A Kongkanand (dr_apichat@yahoo.com)

Received: 1 September 2010; Revised: 13 December 2010; Accepted: 17 January 2011; Published online: 13 June 2011 
these, $\mathrm{PE}$ has recently gained awareness as new treatments have become available. The prevalence of ED in a study of Thai men aged 40-70 years, performed in 1998, was found to be $37.5 \%$ and increased to $42 \%$ in 2003 , as reported by a second survey. ${ }^{6}$ Kongkanand et al. ${ }^{3}$ also identified risk factors in descending order: aging, hypertension, diabetes, cardiovascular problems, smoking, trauma and surgery.

\section{SEXUAL ATTITUDES OF THAI MEN ${ }^{4,5,7-9}$}

In the studies on sexual attitudes, the main risk for ED was attributed to the aging process, whereas risk factors involved in vascular diseases, including diabetes, hypertension and heart disease, were grouped together into a 'bargaining group'. This bargaining group had a characteristic referred to as ' 3 for 100', which meant that if you have one of these three diseases, you would have a calculated ED risk of $75 \%$. If a patient has two diseases, the correlated rate is $85 \%$ and three diseases would translate into a $100 \%$ ED risk. It was also discovered that prostate enlargement also contributed to ED to some degree and that smoking is a major risk factor for ED.

\section{SMOKING}

There is a correlation between smoking and ED, but the key factor is the duration of smoking rather than the number of cigarettes smoked per day. ${ }^{4,5}$ For patients who smoked for more than 30 years, there is a $57 \%$ probability of ED. With regard to severity, those who smoked for 1-15 years only had a $7 \%$ rate of severe $\mathrm{ED}$, but this percentage increases to $20.69 \%$ for patients who have smoked for over 30 years.

\section{EDUCATION}

Education plays an important role in the development of men's health issues later in life. Men who attended or graduated college, or completed some degree of higher education, have improved their health compared to those who did not. ${ }^{4,5}$ Approximately $60 \%$ of men who graduated from primary school have ED, whereas men with secondary school, high school and college or higher education levels have rates of $42 \%, 38 \%$ and $30 \%$, respectively. Education and men's health are likely to be directly related because education improves self-care, healthy diet, exercise and the ability to balance stress and relaxation. ${ }^{5}$

\section{STRESS FACTORS}

In the survey on stress factor, ${ }^{4,5}$ stress played a significant role in men suffering from ED. Although it is not clear whether patients suffer from the stress of ED or if stress precipitates the development of ED, the role of stress is a clear factor in men. Approximately $38 \%$ of men with mild stress have ED and $65 \%$ of men with significant stress have ED.

\section{ALCOHOL}

Moderate alcohol consumption does not impact ED. ${ }^{5}$ However, those who drink heavily and/or suffer from liver cirrhosis may have an increased risk of ED, but that population was not investigated in the study on alcohol consumption. ${ }^{5}$

\section{EXERCISE}

Exercise plays a pivotal role in health and has an impact on quality of daily life. Exercise, which can include attending a fitness program at a gymnasium, jogging, playing tennis or playing golf, can have relaxing properties on the mind and promote physical health. Men who regularly exercise have a lower prevalence of ED (41\%) compared to those who do not (43\%). Moreover, men who exercise regularly also have a lower prevalence of severe ED (10\%), whereas men without regular exercise had a $14 \%$ rate of severe ED.

\section{COUNSELLING}

Counselling for ED has always been a debatable topic. In our studies in $2000,{ }^{4,5}$ we found that most men would seek treatment or recommendations from their wife, partner, friend or physician. Only $32 \%$ of the men sampled refused to discuss ED with anyone and cited embarrassment as the main concern. Among men who discussed ED with their physicians, $72 \%$ felt comfortable discussing ED, whereas only $14 \%$ felt uncomfortable. ${ }^{5}$

\section{PROSTATE CANCER}

Since the development of the prostate-specific antigen in Thailand 15 years ago, transrectal ultrasound and biopsies have become routine practice. ${ }^{10-12}$ As a result of routine examinations, the early detection of prostate cancer has increased significantly, and the incidence of cases is now the fourth highest, following liver, lung and colon cancers. ${ }^{13-17}$ Tantiwong et al., ${ }^{11}$ who surveyed a local Thai community, reported a $41.3 \%$ prevalence of symptomatic $\mathrm{BPH}$ in elderly Thai individuals and an incidence of prostate cancer of $0.75 \%$.

In Thailand, potential treatments include radical prostatectomy, laparoscopy, prostatectomy and robotic prostatectomy. In some institutions, brachytherapy and high-dose radiation therapy are also available. ${ }^{18,19}$

\section{HYPOGONADISM OR LATE-ONSET HYPOGONADISM (LOH) ${ }^{20}$}

There have been recent reports citing metabolic syndrome in the development of ED. Thailand has opened a large number of men's health clinics in addition to clinics within major universities or large community hospitals. These clinics are available to men who are aware of issues related to men's health and include the use of validated Thai-language aging male questionnaires, including the International Prostate Symptom Score and the International Index of Erectile Function-5.

Permpongkosol et al. ${ }^{5}$ performed in a large university hospital (Ramathibodi Hospital, Mahidol University, Bangkok, Thailand) collected data from $42 \%$ of the male patients under their care. The average age at their first visit was 56 years, and most had an education background of a bachelor's degree followed by a master's degree. This reflects the link between higher education and increased health awareness among males. This population consisted of individuals who did not smoke, did not consume alcoholic beverages and approximately $86 \%$ of whom performed regular exercise.

From the study population, there were eight categories of chief complaints, from a total of 20, which included the following: hyperlipidemia, ED, lower urinary tract symptoms (such as BPH, urinary tract infection and prostatitis), $\mathrm{LOH}$, osteopenia, hypertension, diabetes mellitus and osteoporosis. A total of $24 \%$ of all participants were diagnosed with $\mathrm{LOH}$ and had an average age of 57 years.

The questionnaires that have been validated in Thai include the Aging Male Symptom's Score, the International Prostatic Symptom's Score and the International Index of Erectile Function. Metabolic syndrome is diagnosed in males who meet the criteria for metabolic syndrome, defined as central obesity of $>94 \mathrm{~cm}$ (waist circumference) at any time in addition to two of the following: triglycerides $>150 \mathrm{mg} \mathrm{dl}^{-1}$, high-density lipoprotein less than $<40 \mathrm{mg}$ $\mathrm{dl}^{-1}$, blood pressure $>130 / 85 \mathrm{mmHg}$ and FBS $>100 \mathrm{mg} \mathrm{dl}^{-1}$. Of the 80 patients with $\mathrm{LOH}$ in our studies, 35 had metabolic syndrome. The distribution of diseases among the population was metabolic syndrome (43.75\%), ED (63.75\%), hyperlipidemia (68.75\%), hypertension $(42 \%)$, BPH (36\%) and diabetes $(17.5 \%)$. 
In regard to clinic awareness, friends and family were the most common referral method cited by patients, at a rate of $58 \%$, followed by $34 \%$ from TV and newspaper commercials and finally, by physician referrals at $25 \%$. For clinic referrals, individuals who referred patients to the clinic included self-referrals (43\%), friends (23\%), spouses $(26 \%)$ and physicians $(11 \%)$. A majority of patients presenting to the clinic cited the lack of a morning erection, loss of sexual desire, trouble entering deep sleep and fatigue. These patients presented with concomitant medications for BPH (50\%), hyperlipidemia (48\%) and hypertension (37\%).

\section{PE}

$\mathrm{PE}$ is an extremely personal and embarrassing condition that causes an individual to shy away from open discussions with those around him, including his physician. PE is more readily found in young men; however, for men over 40 years of age, PE is closely linked to ED. When this is shared with patients, many reject the diagnosis of ED. Nevertheless, when one insists on the diagnosis and explains that impotence and the inability to sustain an erection results in early ejaculation, patients become more understanding and accepting of the diagnosis. Treatments, including selective serotonin reuptake inhibitors, have been a mainstay for managing PE. In Thailand, we continue to use long-acting selective serotonin reuptake inhibitors for the treatment of PE because newer selective serotonin reuptake inhibitors are not readily available in Thailand.

\section{THE AGING MALE}

Aging males, defined as those 60 years of age or older, account for more than $10 \%$ of the Thai population, or $7-8$ million people. Given the sheer number of males in this category, there is an enormous burden on the government to support the elderly in regard to health care. This is crucial because the number of elderly individuals developing diabetes, hypertension, cardiac diseases, hyperlipidemia, depression and dementia continues to increase. Recently, the government has declared that the country is a socially responsible state that provides free education up to 15 years of age, electrical power, tap water and basic health care.

\section{LOOKING FORWARD TO THE FUTURE}

Thailand is a developing nation that has just crossed the midpoint towards becoming a developed nation. Unfortunately, the political crisis continues to interrupt progression in many ways. It is hoped that Thailand will be able to sidestep these obstacles and continue to develop. Health care in men has significantly improved; however, because men inevitably fall victim to sicknesses as they age, we need competent physicians and adequate hospital services to treat these diseases effectively. Thailand has a high number of government hospitals, but the number of physicians at large community hospitals in major provinces is still short in regard to specialists in trauma, general surgery, neurosurgery and cardiology, despite increased medical graduates totalling 2000 physicians per year.

The current crisis in regard to malpractice compensation, debated between the medical council and consumer groups, has become a hot topic. There is a fear that the impact of this issue will act as a deterrent for potential future medical students, further deepening our need for increased physicians.

\section{ISSUES SURROUNDING NURSING HOMES}

Home care for the elderly, or nursing homes, are in short supply in Thailand because only a few government-run facilities exist, and privately owned nurseries are extremely expensive. Additionally, the degree of personal care being offered is also inadequate, and we would like to see an increase in the quantity and quality of nursing homes in Thailand as compared to other developing countries.

\section{CONCLUSION}

Men's health check-ups, the availability of treatments and the use of keywords are critical success factors for educating men on self-care methods or for managing symptoms and diseases such as prostate enlargement, prostate cancer, ED, PE or hypogonadism. By communicating the importance of risk factors and treatment options, these keywords can provide a stepping stone for self-care and prolonging life expectancy to a level beyond the expectations predicted 20 years ago. Additionally, the development of technological advances in medicine has given physicians the ability to raise the standard of health care to a higher level, further prolonging life expectancy. Overall, ensuring a good quality of life is the ultimate goal.

1 Tsertsvadze A, Yazdi F, Fink HA, MacDonald R, Wilt TJ et al. Oral sildenafil citrate (viagra) for erectile dysfunction: a systematic review and meta-analysis of harms. Urology 2009; 74: 831-6.

2 Langtry HD, Markham A. Sildenafil: a review of its use in erectile dysfunction. Drugs 1999; 57: 967-89.

3 Kongkanand A, Ratana-Olarn K, Ruangdilokrat S, Tantiwong A. The efficacy and safety of oral sildenafil in Thai men with erectile dysfunction: a randomized, double-blind, placebo controlled, flexible-dose study. J Med Assoc Thai 2003; 86: 195-205.

4 Kongkanand A. Prevalence of erectile dysfunction in Thailand. Thai Erectile Dysfunction Epidemiological Study Group. Int J Androl 2000; 23 (Suppl 2): 77-80.

5 An epidemiological study of erectile dysfunction in Thailand (Part 1: Prevalence). Thai Erectile Dysfunction Epidemiologic Study Group (TEDES). J Med Assoc Thai 2000; 83: 872-9.

6 Permpongkosol S, Kongkakand A, Ratana-Olarn K, Tantiwong A, Tantiwongse K. Increased prevalence of erectile dysfunction (ED): results of the second epidemiological study on sexual activity and prevalence of ED in Thai males. Aging Male 2008; 11: 128-33.

7 Guadamuz TE, Kunawararak P, Beyrer C, Pumpaisanchai J, Wei C et al. HIV prevalence, sexual and behavioral correlates among Shan, Hill tribe, and Thai male sex workers in Northern Thailand. AIDS Care 2010; 22: 597-605.

8 Ford K, Chamratrithirong A. First sexual experience and current sexual behaviour among older Thai men and women. Sex Health 2009; 6: 195-202.

9 Rasamimari A, Dancy B, Talashek M, Park CG. Predictors of sexual behaviors among Thai young adults. J Assoc Nurses AIDS Care 2007; 18: 13-21.

10 Tantiwong A, Soontrapa S, Sujijantrarat P, Vanprapar N, Sawangsak L. The prevalence of prostate cancer screening in Thai elderly. J Med Assoc Thai 2002; 85: 502-8.

11 Tantiwong A, Nuanyong C, Vanprapar N, Swasdipala P, Chittapraphai S. Benign prostatic hyperplasia in elderly Thai men in an urban community: the prevalence, natural history and health related behavior. J Med Assoc Thai 2002; 85: 356-60.

12 Leewansangtong S, Tantiwong A, Ratanarapee S, Nualyong C, Soontrapa S. The risks of prostate cancer detection by transrectal ultrasound guide biopsy in Thai men with abnormal prostatic-specific antigen or abnormal digital rectal examination. J Med Assoc Thai 2000; 83: 1519-24.

13 Vatanasapt P, Lertsinudom S, Sookprasert A, Phunmanee A, Pratheepawanit N et al. Prevalence and management of cancer pain in Srinagarind Hospital, Khon Kaen, Thailand. J Med Assoc Thai 2008; 91: 1873-7.

14 Domingo EJ, Noviani R, Noor MR, Ngelangel CA, Limpaphayom KK et al. Epidemiology and prevention of cervical cancer in Indonesia, Malaysia, the Philippines, Thailand and Vietnam. Vaccine 2008; 26 (Suppl 12): M71-9.

15 Khuhaprema T, Srivatanakul P. Colon and rectum cancer in Thailand: an overview. Jpn J Clin Oncol 2008; 38: 237-43.

16 Sriplung H, Wiangnon S, Sontipong S, Sumitsawan Y, Martin N. Cancer incidence trends in Thailand, 1989-2000. Asian Pac J Cancer Prev 2006; 7: 239-44.

17 Vatanasapt V, Sriamporn S, Vatanasapt P. Cancer control in Thailand. Jpn J Clin Oncol 2002; 32 (Suppl 1): S82-91.

18 Leewansangtong S, Soontrapa S, Nualyong C, Srinualnad S, Taweemonkongsap T et al. Is radical prostatectomy in Thai men a high morbidity surgery for localized or locally advanced prostate cancer? J Med Assoc Thai 2005; 88: 1833-7.

19 Leewansangtong S, Soontrapa S, Nualyong C, Srinualnad S, Taweemonkongsap T et al. Outcomes of radical prostatectomy in Thai men with prostate cancer. Asian J Surg 2005; 28: 286-90.

20 Permpongkosol S, Tantirangsee N, Ratana-olarn K. Treatment of 161 men with symptomatic late onset hypogonadism with long-acting parenteral testosterone undecanoate: effects on body composition, lipids, and psychosexual complaints. J Sex Med 2010; 7: 3765-74. 